\title{
中空核壳结构 $\alpha-\mathrm{MoO}_{3}-\mathrm{SnO}_{2}$ 复合电极可控制备及 储锂性能研究
}

\author{
王亚朋，刘佳佳，刘春晓，陈伟伟，李婷婷，郭 洪 \\ (云南大学 化学科学与工程学院, 昆明 650031)
}

摘 要: 采用醇热技术可控制备了中空核壳结构 $\alpha-\mathrm{MoO}_{3}-\mathrm{SnO}_{2}$ 二次锂离子电池复合负极材料。通过 XRD、SEM、 TEM、CV 和恒流充放电等测试手段对材料结构、形貌和电化学性能进行了表征。结果表明: 构建的多元金属氧化 物既具有电化学活性成分, 又含有骨架支撑部分, 独特的中空结构有效地缩短了电子和锂离子传输路径。电化学测 试表明：该材料在电流密度 $50 \mathrm{~mA} / \mathrm{g}$ 时循环 100 次后放电比容量仍高达 $865 \mathrm{mAh} / \mathrm{g}$ 。在电流密度为 $1000 \mathrm{~mA} / \mathrm{g}$ 时循 环 100 次后放电比容量仍达到 $545 \mathrm{mAh} / \mathrm{g}$, 表现出优异的循环性能和倍率性能。该合成方法简单、成本低, 产量高, 可为制备其它中空核壳结构先进功能材料提供借鉴。

关 键 词: 二氧化锡; 三氧化锄; 中空核壳材料; 纳米结构; 电化学

中图分类号: TQ174 文献标识码: A

\section{Morphology-controlled Synthesis of Hollow Core-shell Structural $\alpha-\mathrm{MoO}_{3}-\mathrm{SnO}_{2}$ with Superior Lithium Storage}

\author{
WANG Ya-Peng, LIU Jia-Jia, LIU Chun-Xiao, CHEN Wei-Wei, LI Ting-Ting, GUO Hong \\ (School of Chemical Science and Technology, Yunnan University, Kunming 650091, China)
}

\begin{abstract}
An effective approach of alcoholysis was employed to prepare hollow core-shell $\mathrm{MoO}_{3}-\mathrm{SnO}_{2}$ hybrid nanoparticle aggregates as anode materials for Li-ion batteries. The as-prepared samples were characterized by XRD, SEM, TEM, CV, and galvanostatical method. The results show that the unique hollow structures can shorten the distance Li-ion diffusion, and the hollow structure offers a sufficient void space, which sufficiently alleviates the mechanical stress caused by volume change. The as-obtained hollow core-shell $\mathrm{MoO}_{3}-\mathrm{SnO}_{2}$ hybrid electrodes can retain a discharge capacity of $865 \mathrm{mAh} / \mathrm{g}$ at current density of $50 \mathrm{~mA} / \mathrm{g}$ after 100 charge-discharge cycles. Even at the current density of $1000 \mathrm{~mA} / \mathrm{g}$, the $\mathrm{MoO}_{3}-\mathrm{SnO}_{2}$ hybrid electrodes can still deliver a high reversible discharge capacity of $545 \mathrm{mAh} / \mathrm{g}$. Therefore, the hollow $\mathrm{MoO}_{3}-\mathrm{SnO}_{2}$ hybrid electrode exhibits stable cyclability and good rate capability. This method is simple, low cost and mass-productive, and may also be used to prepare other advanced functional materials.
\end{abstract}

Key words: $\mathrm{SnO}_{2} ; \mathrm{MoO}_{3}$; hollow core-shell materials; nanostructures; electrochemistry

2008 年, Tarascon 小组研究发现纳米过渡金属 氧化物 $(\mathrm{Mo} 、 \mathrm{Ni} 、 \mathrm{Co} 、 \mathrm{Mo} 、 \mathrm{Cu})$ 具有较高的质量比
容量(700 900 mAh/g)和较好的倍率性能, 远高于目 前商用石墨的比容量 $(372 \mathrm{mAh} / \mathrm{g})$, 其体积比容量高 
达 3500 4500 mAh/mL $\mathrm{mL}^{[1]}$ 。同时, 它们的电位高于金 属锂, 可以防止锂枝晶的产生, 提高了电池的安全 性能。并且, 它们的来源广泛, 成本较低, 为工业规 模化生产提供了基本保障。另外, 该类氧化物存在 可变的化合价和多样的物相结构, 不同条件下可以 合成形貌独特、成分各异的纳微结构 ${ }^{[2-4]}$ 。但是, 该 类材料依然存在脱嵌锂过程缓慢、体积效应大、倍 率性能差、首次充放电库仑效率低等问题 ${ }^{[5]}$, 主要原 因在于 $3 \mathrm{~d}$ 过渡金属电极材料反应机理不同于传统 的插层机理和合金机理, 而是在放电过程中氧化物 与金属锂反应, 生成氧化锂和金属单质; 在充电过 程中氧化锂与金属单质反应重新生成过渡金属氧化 物和金属锂。过渡金属氧化物多数是导电性较差的 半导体材料, 在反应过程中生成的 $\mathrm{Li}_{2} \mathrm{O}$ 进一步恶化 了材料的导电性 ${ }^{[4]}$ 。同时, 过渡金属氧化物颗粒首次 嵌锂后, 往往会发生较大的体积膨胀, 会导致颗粒 间及颗粒与集流体间在循环过程中逐渐失去电接触 [6]。此外, 锂离子嵌入后, 纳米颗粒表面的原子相互 成键而使近邻的纳米颗粒逐渐融合, 从而引发严重 的电化学团聚, 丧失电化学活性 ${ }^{[7-8]}$ 。因此, 开发比 容量高、循环性能和倍率性能好的过渡金属基负极 材料面临很大挑战。

构建多元复合过渡金属氧化物是提高其循环性 能的一个重要手段 ${ }^{[9-11]}$ 。多元复合电极材料既有电 化学活性成分, 又具有骨架支撑, 可以有效提高锂 离子电池的循环性能和倍率性能。同时, 辅助物在 复合材料中可以提高活性物质的电子导电性。另外, 不同组分的嵌锂电位略有不同, 一相参与反应时, 另外一相可充当惰性介质的作用, 也可缓冲充放电 过程中电极的体积膨胀 ${ }^{[12-14]}$ 。

设计合理的纳米结构是改善过渡金属氧化电极 材料电化学性能的另一重要手段 ${ }^{[15-18]}$ 。而传统的纳 米颗粒虽然在一定程度上对电极的循环性能有所改 善，但在制备中易带入大量的表面杂质，导致首次 充放电效率较低, 特别是循环过程中的剧烈团聚, 限制了其循环性能的进一步提高 ${ }^{[19]}$ 。因此, 仅仅减 小材料的粒度并不能完全改善多元过渡金属类电极 的循环性能。文献[20-21]研究显示, 分级中空及中 空核壳结构表现出更为优异的结构稳定性和电化学 活性。本课题组前期制备的空心核壳结构 $\mathrm{SnO}_{2} @ \mathrm{C}$ 材料具有高储锂性能 ${ }^{[22]}$ 。Lou 课题组 ${ }^{[23]}$ 合成的的 $\mathrm{Fe} 、 \mathrm{~V}$ 中空氧化物; Hou 等 ${ }^{[3]}$ 合成的 Co、Mn 空心氧 化物; SUN 等 ${ }^{[24]}$ 合成的 Mo 中空氧化物和 Wang 等 ${ }^{[25]}$ 合成的 IVA 族元素中空材料的电化学性能都明显优 于普通纳米颗粒。因此, 本研究结合多元复合材料
和中空纳米结构的优势, 以 Mo-Sn-O 为对象, 探索 分级中空纳米结构混合过渡金属氧化物可控制备, 并分析合成材料的储锂性能。

\section{1 实验方法}

\section{1 中空核壳结构 $\mathrm{MoO}_{3}-\mathrm{SnO}_{2}$ 纳米材料的制备}

实心、中空核壳及空心结构电极材料均由改性 的醇热反应得到。将 $\mathrm{SnCl}_{2} \cdot 2 \mathrm{H}_{2} \mathrm{O}(0.54 \mathrm{~g})$, $\left(\mathrm{NH}_{4}\right)_{6} \mathrm{Mo}_{7} \mathrm{O}_{24} \cdot 4 \mathrm{H}_{2} \mathrm{O}(2.76 \mathrm{~g})$ 和葡萄糖 $(1.0 \mathrm{~g})$, 溶于 乙醇 $(35 \mathrm{~mL})$ 和甘油 $(15 \mathrm{~mL})$ 的混合物中, $180^{\circ} \mathrm{C}$ 下 反应 6 48 h, 将反应所得沉淀离心分离, 用去离子 水和无水乙醇反复洗涤, 所得产品在 $60^{\circ} \mathrm{C}$ 下真空干 燥 $24 \mathrm{~h}$ 。最后在马弗炉中 $400^{\circ} \mathrm{C}$ 加热 $2 \mathrm{~h}$, 除去水和 杂质, 可得到深绿色 $\alpha-\mathrm{MoO}_{3}-\mathrm{SnO}_{2}$ 粉末。

\section{2 样品的表征}

采用 D/Max-3B 型 X 射线衍射仪(日本)进行物 相分析, S-360 型扫描电子显微镜(日本) 观察样品 的颗粒形貌, JEM-2100 型透射电子显微镜(日本)进 一步观察样品的微观结构, ASAP-2000 型氮气吸附 测量仪(美国)测定样品的比表面积。

\section{3 电化学测试}

按质量比将 $75 \%$ 的活性材料、 $15 \%$ 的导电剂乙 炔黑和 10\%粘结剂 PVDF 的混合物溶于 $\mathrm{N}$-甲基吡咯 烷酮, 然后在玛瑙研钵中将混合物研磨成均匀的浆 料, 涂在干净的铜箔上制成电极片。将电极片置于 干燥箱中 $50^{\circ} \mathrm{C}$ 预干燥, 待 $\mathrm{N}$-甲基吡咯烷酮完全挥 发后, 在压机上轻压, 用打孔器将完全干燥的膜卡 成 $\phi 1.5 \mathrm{~cm} \times 50 \mu \mathrm{m}$ 的电极片, 在 $120^{\circ} \mathrm{C}$ 条件下真空 干燥 $24 \mathrm{~h}$ 备用。用锂片作对电极, $1 \mathrm{~mol} / \mathrm{L} \mathrm{LiPF}_{6} / \mathrm{EC}+$ $\mathrm{DMC}($ 体积比 $1: 1)$ 作为电解液, 在充满氩气的手套 箱内组装成实验电池。采用上海辰华公司生产的 CHI660D 电化学工作站进行循环伏安测试。采用武 汉蓝电电池测试系统 LAND2100, 对组装的电池进 行恒电流充放电实验, 电压范围 $0.01 \sim 1.5 \mathrm{~V}$ 。

\section{2 结果与讨论}

\section{1 物性结构及合成机制分析}

图 1(A)为产品及其前驱体的 $\mathrm{X}$ 射线衍射图谱, 表明合成材料为 $\alpha-\mathrm{MoO}_{3}\left(\mathrm{JCPDS}\right.$ 65-2421)和 $\mathrm{SnO}_{2}$ (JCPDS 41-1445)复合物。相对较高的背底显示存在 无定形结构。根据热重(TG)分析显示产品中仍含有 $2.5 \mathrm{wt} \%$ 的碳。通过谢乐公式计算 $\mathrm{MoO}_{3}$ 的平均晶粒 度约为 $8 \mathrm{~nm}$, 表明产品由纳米晶体单元组成。图 1(B) 

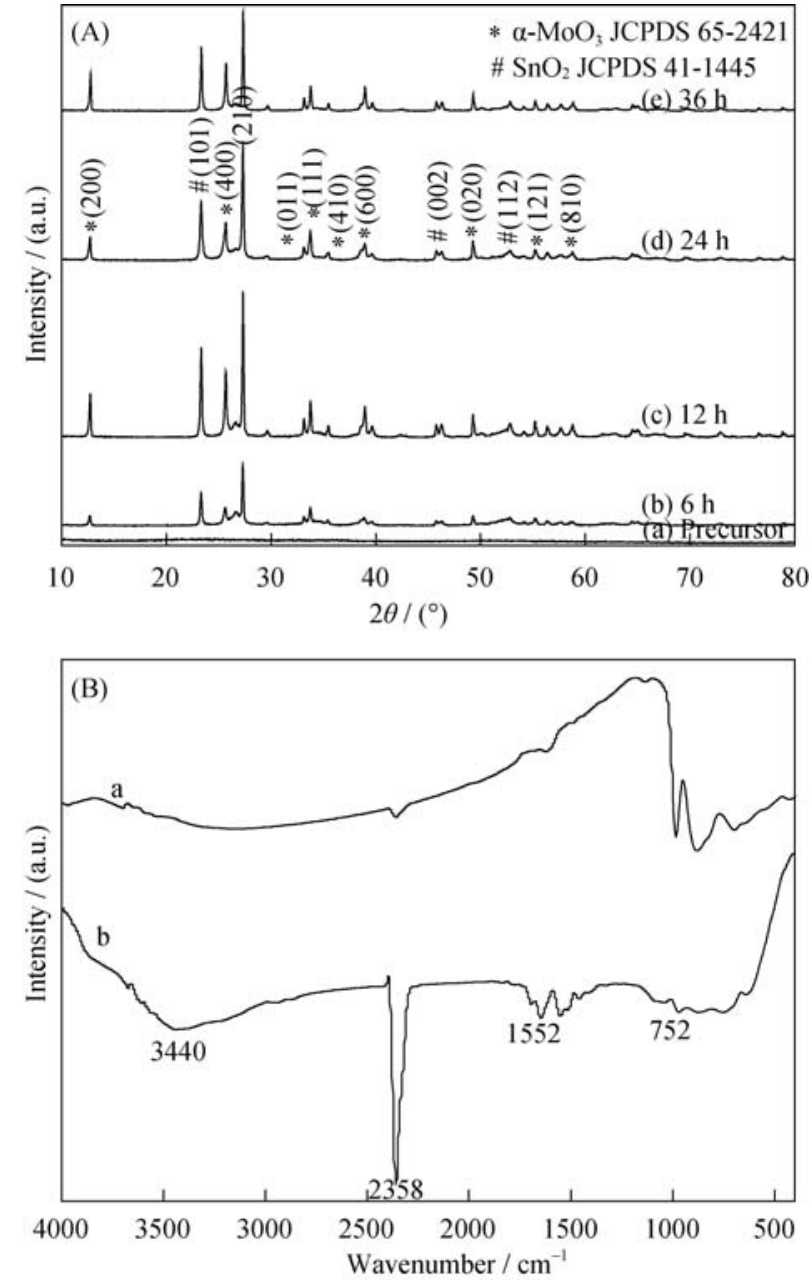

图 1 不同醇热反应时间(6 36 h) 合成 Mo-Sn-O 产品及前驱体 的 XRD 图谱(A)和醇热反应的前驱体及 $400^{\circ} \mathrm{C}$ 炦烧后样品的 红外光谱图(B)(其中, 曲线 $\mathrm{a}$ 为醇热反应 $24 \mathrm{~h}$ 的样品, 曲线 $\mathrm{b}$ 为其前驱体)

Fig. 1 (A) XRD pattern of Mo-Sn-O samples prepared by alcoholysis synthesis process and subsequent calcination at $400{ }^{\circ} \mathrm{C}$, (B) FTIR spectra of the sample and its precursor, and the curve $\mathrm{a}$ and $\mathrm{b}$ corresponding to the Mo-Sn-O sample alcoholysis synthesis for $24 \mathrm{~h}$ and calcined at $400^{\circ} \mathrm{C}$ and the precursor, respectively

为中空核壳 $\mathrm{MoO}_{3}-\mathrm{SnO}_{2}$ 材料及其前驱体的红外光 谱图。集中在 $3440 \mathrm{~cm}^{-1}$ 和 $2358 \mathrm{~cm}^{-1}$ 的峰位对应吸 收水分子的 $-\mathrm{OH}$ 非对称和对称伸缩振动; $1552 \mathrm{~cm}^{-1}$ 峰对应 $\mathrm{MoO}_{3}-\mathrm{SnO}_{2}$ 粒子表面氢氧根的水分子的弯 曲振动峰。500 1000 $\mathrm{cm}^{-1}$ 之间的宽吸收峰对应金属 $-\mathrm{O}$ 键, 其峰值强度不同于前驱体, 表明所制备的样 品的结构与前驱体有差异。

图 2(a) (c)为合成样品(醇热反应 $24 \mathrm{~h}, 400^{\circ} \mathrm{C}$ 煅 烧)在不同放大倍数下的 SEM 照片。合成样品为平均 粒径约 $2 \mu \mathrm{m}$ 的中空核壳结构均匀球体, 如图 2(a)和(b) 所示。根据图 2(c), 壳层的厚度大约 $80 \mathrm{~nm}$ 。根据 TEM 和 HRTEM 分析(图 3(d) (f)), 表面由 $4 \mathrm{~nm}$ 的薄膜
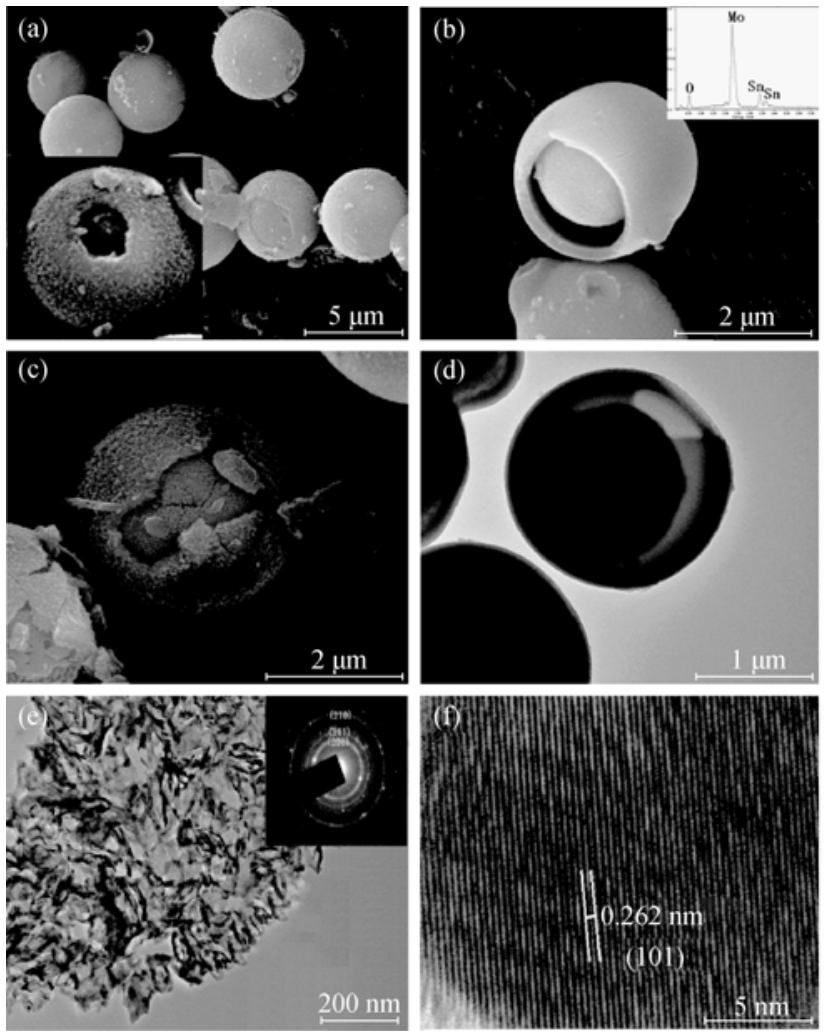

图 2 醇热反应 $24 \mathrm{~h}$, 并经 $400^{\circ} \mathrm{C}$ 煅烧样品的 SEM(a c ), TEM (d e) 和 HRTEM(f)照片

Fig. 2 SEM (a-c), TEM (d-e) and HRTEM (f) images of MoSn-O sample prepared by alcoholysis syntheses for $24 \mathrm{~h}$ and subsequent calcination at $400{ }^{\circ} \mathrm{C}$

组装而成。根据选定区域的电子衍射(SAED)花样分析, 衍射环分别与 $\alpha-\mathrm{MoO}_{3}$ 的(200)、(211)和(210)晶面匹 配。样品的 HRTEM 图片显示晶格间距 $(0.26 \mathrm{~nm})$, 与 $\mathrm{SnO}_{2}(101)$ 晶面间距相吻合, 这与 XRD 和 EDX 的分析 结果一致。图 3 是中空核壳 $\mathrm{MoO}_{3}-\mathrm{SnO}_{2}$ 纳米材料的

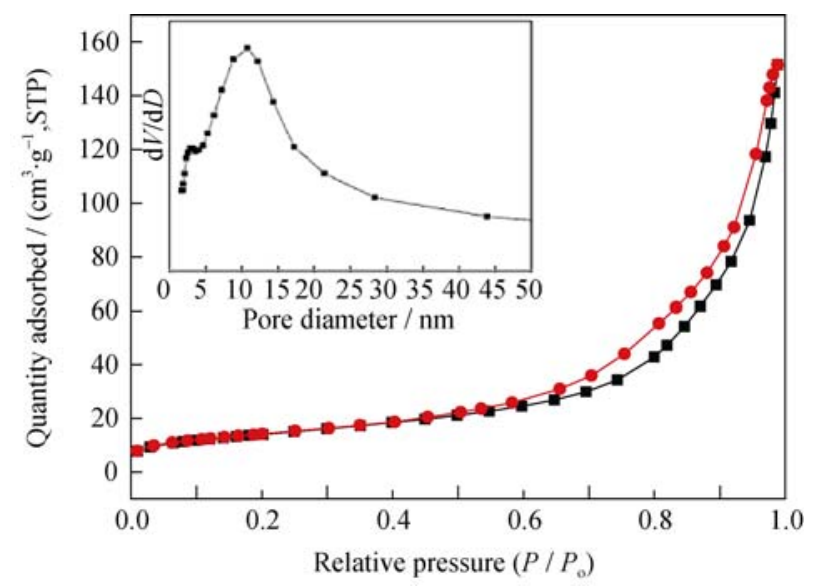

图 3 醇热反应 $24 \mathrm{~h}$, 并经 $400^{\circ} \mathrm{C}$ 敾烧样品的氮气吸附/脱附等 温线和 Barrett-Joyner-Halenda(BJH) 孔径分布图

Fig. 3 Nitrogen adsorption/desorption isotherm and BarrettJoyner-Halenda (BJH) pore size distribution plot (inset) of the Mo-Sn-O samples prepared by alcoholysis syntheses for $24 \mathrm{~h}$ and subsequent calcination at $400{ }^{\circ} \mathrm{C}$ 
$\mathrm{N}_{2}$ 吸附/脱附等温线和孔径分布图。样品的比表面积 为 $95.25 \mathrm{~m}^{2} / \mathrm{g}$ 。这种介孔结构不仅能保持活性成分 的纳米效应，也可缓冲 $\mathrm{MoO}_{3}-\mathrm{SnO}_{2}$ 电极在充放电 过程中的体积变化, 从而改善电极材料的电化学 性能。

图 4 为 $180^{\circ} \mathrm{C}$ 下，醇热反应 $6 、 12 、 18 、 30 、 36$ 和 $48 \mathrm{~h}$ 所得样品的 SEM 照片。与图 2 相比, 醇热 反应得到的产品接近于㷽烧后生成的最终产品。醇 热反应 $6 \mathrm{~h}$, 样品为实心结构(图 4(a))。醇热反应 $12 \mathrm{~h}$ (图 4(b)), 样品表面逐渐粗糙, 并形成中空核壳结 构。随着醇热反应时间的延长(图 4(c), $18 \mathrm{~h}$ ), 核壳 结构更加明显。进一步延长醇热反应时间, 球的内 部逐渐变空(图 4(d), $30 \mathrm{~h}$ )。最后, 中空核壳结构破 裂, 如图 4(e)(36 h)和 4(f) (48 h)。根据上述分析, $\mathrm{MoO}_{3}-\mathrm{SnO}_{2}$ 中空核壳的生成是基于奥斯特瓦尔德 熟化理论(如示意图 1), 醇热过程中生成的少量水 促使 $\mathrm{Mo}^{6+}$ 和 $\mathrm{Sn}^{2+}$ 水解, 导致 $\mathrm{MoO}_{3}-\mathrm{SnO}_{2}$ 生成和晶 化。但如果向乙醇和甘油体系中加入一定量的水只 能得到固体球，这实际上是由于 $\mathrm{Mo}^{6+}$ 和 $\mathrm{Sn}^{2+}$ 的水 解速度较快, 与葡萄糖的碳化不同步, 因此,
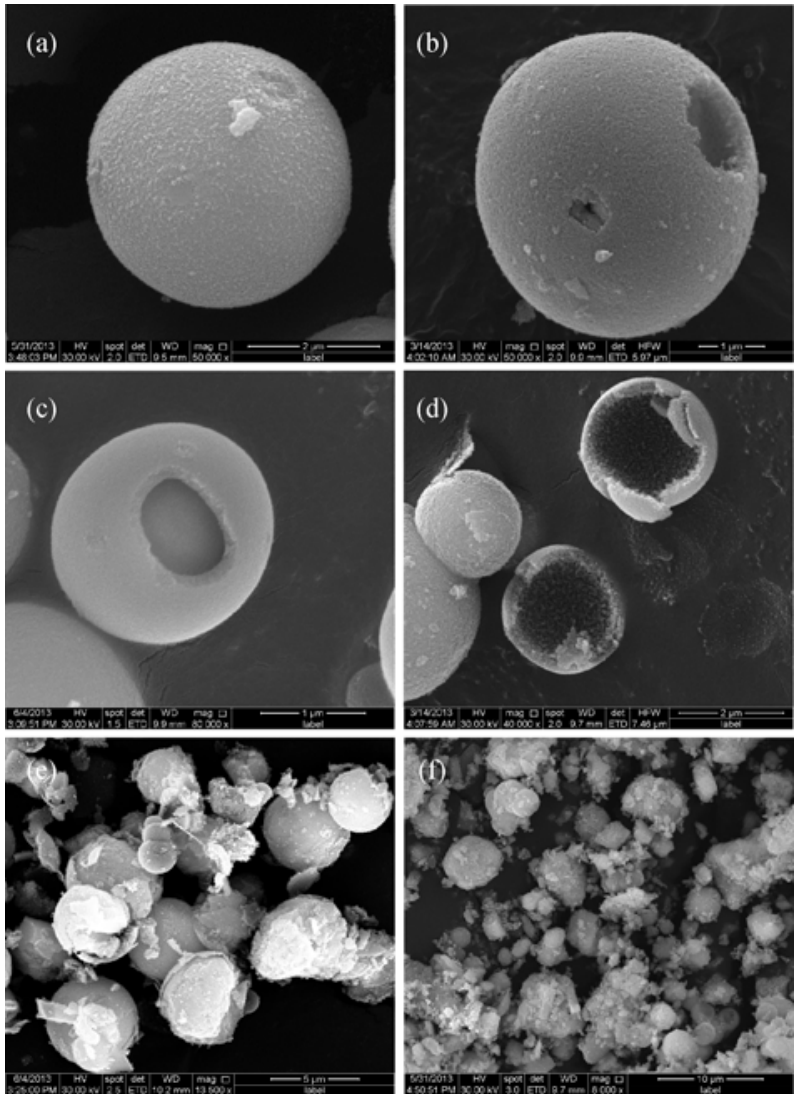

图 4 醇热 $\left(180{ }^{\circ} \mathrm{C}\right)$ 反应 $6 \mathrm{~h}(\mathrm{a}) 、 12 \mathrm{~h}(\mathrm{~b}) 、 18 \mathrm{~h}(\mathrm{c}) 、 30 \mathrm{~h}(\mathrm{~d})$ 、 $36 \mathrm{~h}(\mathrm{e})$ 和 $48 \mathrm{~h}$ (f) 所得 Mo-Sn-O 样品的 SEM 照片

Fig. 4 SEM images of Mo-Sn-O samples after solvothermal alcoholysis at $180{ }^{\circ} \mathrm{C}$ for $6 \mathrm{~h}$ (a), $12 \mathrm{~h} \mathrm{(b),} 18 \mathrm{~h} \mathrm{(c),} 30 \mathrm{~h} \mathrm{(d),} 36 \mathrm{~h}$ (e) and $48 \mathrm{~h}(\mathrm{f})$

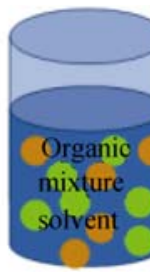

$\mathrm{Sn}^{2+}$

$\mathrm{Mo}^{2+}$
Self-gathering

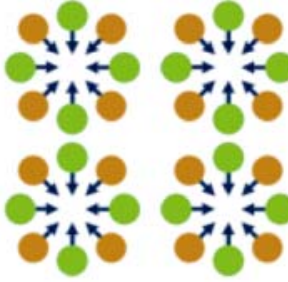

Solvothermal alcoholysis
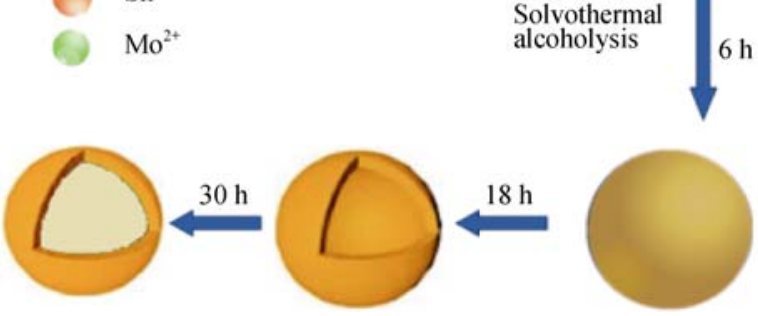

示意图 1 中空核壳结构 Mo-Sn-O 混合纳米材料形成过程

Scheme 1 Illustration of core-shell Mo-Sn-O@C hybrid nanoparticle aggregates synthesized by solvothermal alcoholysis

$\mathrm{MoO}_{3}-\mathrm{SnO}_{2}$ 纳米颗粒只能包覆上无定形碳。与 $\mathrm{Lou}$ 课题组 ${ }^{[4]}$ 报道的方法相比，我们采用的新䓉醇热 制备方法所得产品产量大，且成本低，便于规模 化生产。

\section{2 电化学性能研究}

对所制备的 $\mathrm{MoO}_{3}-\mathrm{SnO}_{2}$ 锂电池负极材料进行电 化学性能研究。图 5(a)显示电极的性能与合成样品 的醇热反应时间有密切关系，醇热反应时间从 $6 \mathrm{~h}$ 增加到 $24 \mathrm{~h}$, 所制备电极的循环稳定性和容量也相 应增高。但是醇热反应时间 $48 \mathrm{~h}$ 合成电极的性能明 显衰减。主要原因在于随着醇热反应时间的延长, 样品的中空核壳结构逐渐被破坏。图 5(b) 为 $\mathrm{MoO}_{3}-\mathrm{SnO}_{2}$ 纳米颗粒(醇热反应 $24 \mathrm{~h}$ ) 的充放电循环 曲线。从图中可以看出, 阴极峰集中在 $0.73 \mathrm{~V}$, 这与 SEI 膜的形成有关, 阳极峰值集中在 $1.42 \mathrm{~V}$ 可以认 为是锂离子从氧化物中脱出的过程。这些结果与其 他课题组的结果相一致 ${ }^{[26-27]}$ 。图 5(c) 是在恒定电流 密度分别为 $50 、 500$ 和 $1000 \mathrm{~mA} / \mathrm{g}$ 时, $\mathrm{MoO}_{3}-\mathrm{SnO}_{2}$ 电 极 $(2 \mathrm{~h})$ 的电化学循环性能。电极的稳定可逆容量为 $865 \mathrm{mAh} / \mathrm{g}(50 \mathrm{~mA} / \mathrm{g}$ 的电流密度下), 100 次循环后仍 可保持在 $807 \mathrm{mAh} / \mathrm{g}$, 可循环率达 $93.1 \%$ 。除了首次 充放电, 库仑效率一直保持在 $99.4 \%$ 以上。初始容 量损失仅为 $120 \mathrm{mAh} / \mathrm{g}$, 这可归结于 SEI 膜的形成 以及 $\mathrm{MoO}_{3}-\mathrm{SnO}_{2}$ 还原为 $\mathrm{Sn} 、 \mathrm{Mo}$ 和 $\mathrm{Li}_{2} \mathrm{O}$ 。初始库仑 效率为 $87.7 \%$ ，高于大多数报道的 $\mathrm{MoO}_{3}$ 和 $\mathrm{SnO}_{2}$ 电 极的效率 ${ }^{[26-27]}$ 。为了研究电极的高倍率性能, 在 500 和 $1000 \mathrm{~mA} / \mathrm{g}$ 电流密度下进行恒电流充放电测试。 电流密度为 $500 \mathrm{~mA} / \mathrm{g}$ 时, 电极材料的放电容量在 717 660 $\mathrm{mAh} / \mathrm{g}$, 表现出良好的稳定性, 库伦效率 保持在 $99.2 \%$ 以上; 电流密度为 $1000 \mathrm{~mA} / \mathrm{g}$ 时, 放电 

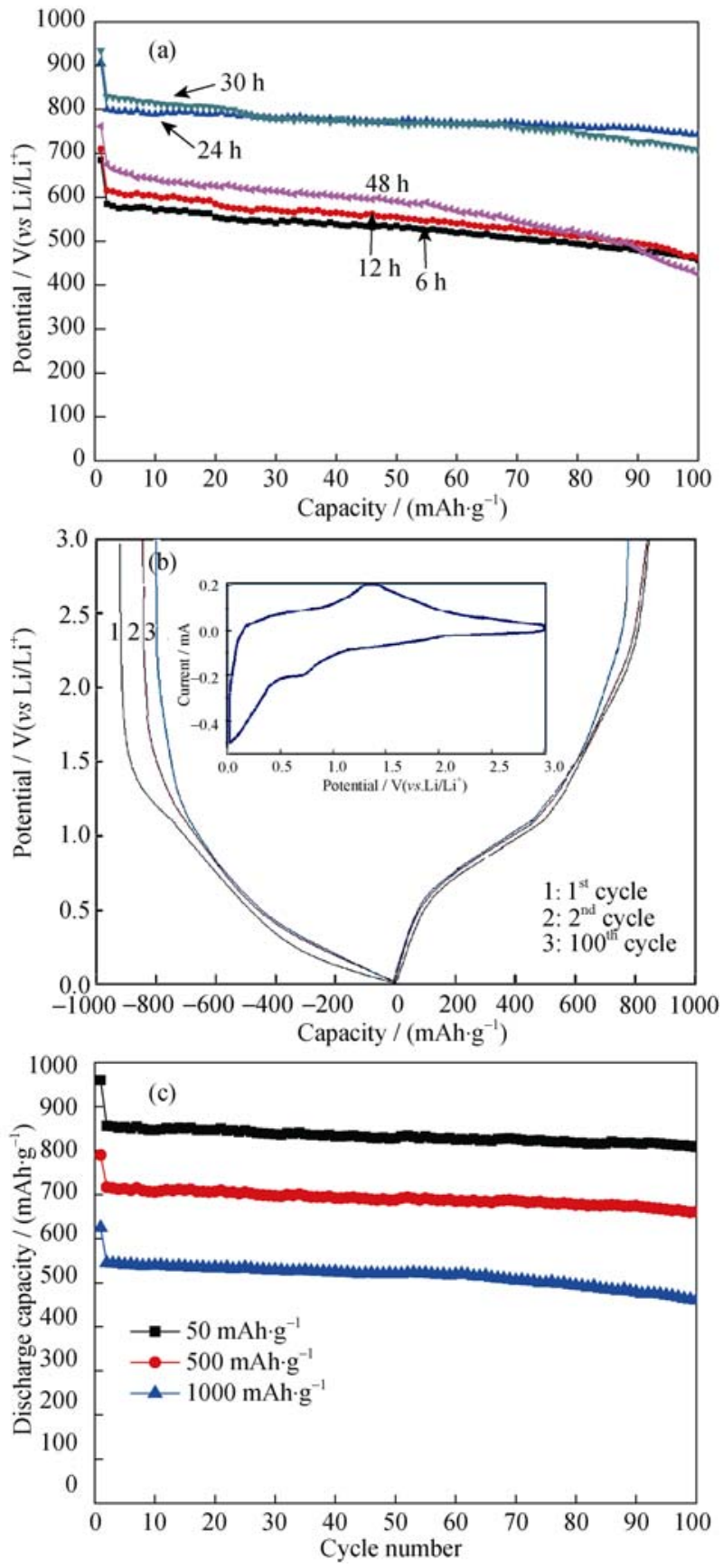

图 5 Mo-Sn-O 材料电极的电化学性能

Fig. 5 Electrochemical performance of the prepared Mo-Sn-O materials electrode

(a) Cycling performance of Mo-Sn-O materials prepared with different solvothermal alcoholysis time from $6 \mathrm{~h}$ to $48 \mathrm{~h}$ at constant current density of $200 \mathrm{~mA} / \mathrm{g}$; (b) charge/discharge curves of Mo-Sn-O (24 h) electrode for the $1^{\text {st }}, 2^{\text {nd }}$, and $100^{\text {th }}$ cycle at current density of $200 \mathrm{~mA} / \mathrm{g}$. The inset in (b) is the first cycle CV curve of f Mo-Sn-O (24 h) electrode with a scan rate of $0.05 \mathrm{mV} / \mathrm{s}$; (c) Cycling performance of Mo-Sn-O $(24 \mathrm{~h})$ electrode at different current densities o. Electrode potential range of $0.01-3.0 \mathrm{~V}\left(v s \mathrm{Li} / \mathrm{Li}^{+}\right)$

容量为 545 468 mAh/g, 库伦效率超过 $98.7 \%$ 。尽管 在高电流密度下比容量有所降低, 但产品的循环性 能仍然保持稳定。图 6 是混合中空 Mo-Sn-O 电极在 恒定电流密度为 $500 \mathrm{~mA} / \mathrm{g}$ 下循环 100 次后的 $\mathrm{TEM}$

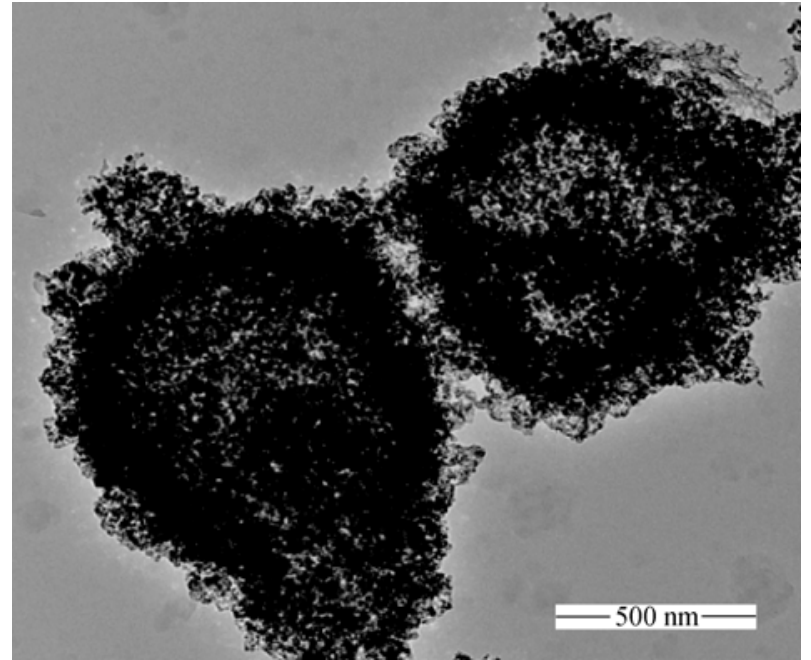

图 6 恒定电流密度为 $500 \mathrm{~mA} / \mathrm{g}$, 充放电循环 100 次后中空 核壳结构 Mo-Sn-O 电极的 TEM 照片

Fig. 6 TEM image of hybrid hollow Mo-Sn-O hybrid electrodes after 100 charge-discharge cycles at current density of $500 \mathrm{~mA} / \mathrm{g}$

形貌，表明材料的中空结构在充放电的过程仍保持 良好，无明显破损。与之前合成 $\mathrm{MoO}_{x}$ 和核壳结构 $\mathrm{SnO}_{2}$ 材料的报道相比 ${ }^{[4,28]}$, 该合成方法简单经济、 环境友好, 并且电化学性得到明显改善。

\section{3 结论}

本研究以改性的醇热技术制备出中空核壳结构 $\alpha-\mathrm{MoO}_{3}-\mathrm{SnO}_{2}$ 复合纳米材料。复合材料在电流密度 $50 \mathrm{~mA} / \mathrm{g}$ 时进行循环, 放电容量一直能够维持在 $850 \mathrm{mAh} / \mathrm{g}$ 左右, 初始容量损失仅为 $120 \mathrm{mAh} / \mathrm{g}$ 。 除了首次充放电，库仑效率保持在 $99.4 \%$ 以上。当 倍率提高到 $500 \mathrm{~mA} / \mathrm{g}$ 和 $1000 \mathrm{~mA} / \mathrm{g}$ 时，材料的稳定 放电容量分别可达 714 和 $545 \mathrm{mAh} / \mathrm{g}$, 表现出极好 的容量保持率和倍率性能。 $\mathrm{MoO}_{3}-\mathrm{SnO}_{2}$ 的优异电化 学性能与其特殊的结构密不可分。一方面, 多元金 属氧化物既具有电化学活性成分, 又含有骨架作用 部分，改善了锂离子电池的循环稳定性。另一方面， 独特的中空核壳结构为脱、嵌锂过程中的体积膨胀 提供了足够的空间, 并且缩短了锂离子扩散路径, 使电极具有高比容量和良好的倍率性能。

\section{参考文献:}

[1] BRUCE P G, SCROSATI B, TARASCON J M, et al. Nanomaterials for rechargeable lithium batteries. Angewandte Chemie International Edition, 2008, 47(16): 2930-2946.

[2] WANG B, CHEN J S, WU H B, et al. Quasiemulsion-templated formation of $\alpha-\mathrm{Fe}_{2} \mathrm{O}_{3}$ hollow spheres with enhanced lithium stor- 
age properties. Journal of the American Chemical Society, 2011, 133(43): 17146-17148.

[3] YUAN C, ZHANG L, HOU L, et al. Green template-free synthesis of mesoporous ternary $\mathrm{CoNi}-\mathrm{Mn}$ oxide nanowires towards high-performance electrochemical capacitors. Particle \& Particle Systems Characterization, 2014, 31(7): 778-787.

[4] LOU X W, LI C M, ARCHER L A. Designed synthesis of coaxial $\mathrm{SnO}_{2} @$ carbon hollow nanospheres for highly reversible lithium storage. Advanced Materials, 2009, 21(24): 2536-2539.

[5] NITTA N, YUSHIN G. High-capacity anode materials for lithiumion batteries: choice of elements and structures for active particles. Particle \& Particle Systems Characterization, 2014, 31(3): 317-336.

[6] YIN Y, RIOUX R M, ERDONMEZ C K, et al. Formation of hollow nanocrystals through the nanoscale Kirkendall effect. Science, 2004, 304(5671): 711-714.

[7] ANUMOL E, NETHRAVATHI C, RAVISHANKAR N. Mechanistic insights into a non-classical diffusion pathway for the formation of hollow intermetallics: a route to multicomponent hollow structures. Particle \& Particle Systems Characterization, 2013, 30(7): 590-598.

[8] LIU L, GUO Y, WANG Y, et al. Hollow NiO nanotubes synthesized by bio-templates as the high performance anode materials of lithium-ion batteries. Electrochimica Acta, 2013, 114: 42-47.

[9] YUAN C, LI J, HOU L, et al. Template-free fabrication of mesoporous hollow $\mathrm{ZnMn}_{2} \mathrm{O}_{4}$ sub-microspheres with enhanced lithium storage capability towards high-performance Li-ion batteries. Particle \& Particle Systems Characterization, 2014, 31(6): 613.

[10] AZIZI A, KHOSLA T, MITCHELL B S, et al. Tuning carbon content and morphology of $\mathrm{FeCo} /$ graphitic carbon core-shell nanoparticles using a sal-matrix-assisted CVD process. Particle \& Particle Systems Characterization, 2014, 31(4): 474-480.

[11] GAO J, MU X, LI J J, et al. Preparation and characterization of porous spherical $\mathrm{Li}_{4} \mathrm{Ti}_{5} \mathrm{O}_{12} / \mathrm{C}$ anode material for lithium batteries. Journal of Inorganic Materials, 2012, 27(3): 253-257.

[12] GUO H, WANG Y, WANG W, et al. Template-free fabrication of hollow NiO-carbon hybrid nanoparticle aggregates with improved lithium storage. Particle \& Particle Systems Characterization, 2014, 31(3): 374-381.

[13] LIU L, YUAN Z Z, QIU C X, et al. Synthesis and electrochemical characteristics of the novel $\mathrm{FeS}_{2} / \mathrm{VGCF}$ material for lithium-ion batteries. Journal of Inorganic Materials, 2013, 28(12): 12911295.

[14] LIU S, ZHANG N, XU Y. Core-shell structured nanocomposites for photocatalytic selective organic transformations. Particle \& Particle Systems Characterization, 2014, 31(5): 540-556.
[15] BAO Y, YANG Y Q, MA J Z. Research progress of hollow structural materials prepared via templating method. Journal of Inorganic Materials, 2013, 28(5): 459-468.

[16] GUO H, HE Y, WANG Y, et al. Morphology-controlled synthesis of cage-bell $\mathrm{Pd} @ \mathrm{CeO}_{2}$ structured nanoparticle aggregates as catalysts for the low-temperature oxidation of CO. Journal of Materials Chemistry A, 2013, 1(25): 7494-7499.

[17] GUO H, WANG W, LIU L, et al. Shape-controlled synthesis of $\mathrm{Ag} @ \mathrm{TiO}_{2}$ cage-bell hybrid structure with enhanced photocatalytic activity and superior lithium storage. Green Chemistry, 2013, 15(10): 2810-2816.

[18] DENG J, YAN C, YANG L, et al. Sandwich-stacked $\mathrm{SnO}_{2} / \mathrm{Cu}$ hybrid nanosheets as multichannel anodes for lithium ion batteries. ACS Nano, 2013, 7(8): 6948-6954.

[19] SCHILLING C, THEISSMANN R, NOTTHOFF C, et al. Synthesis of small hollow $\mathrm{ZnO}$ nanospheres from the gas phase. Particle \& Particle Systems Characterization, 2013, 30(5): 434-437.

[20] XIN S, YIN Y, WAN L, et al. Batteries: encapsulation of sulfur in a hollow porous carbon substrate for superior Li-S batteries with long lifespan. Particle \& Particle Systems Characterization, 2013, 30(4): 392.

[21] GUO H, LIU L, LI T, et al. Accurate hierarchical control of hollow crossed $\mathrm{NiCo}_{2} \mathrm{O}_{4}$ nanocubes for superior lithium storage. Nanoscale, 2014, 6: 5491-5497.

[22] GUO H, MAO R, YANG X, et al. Hollow nanotubular $\mathrm{SnO}_{2}$ with improved lithium storage. Journal of Power Sources, 2012, 219: 280-284.

[23] LOU X W, ARCHER L A. A general route to nonspherical anatase $\mathrm{TiO}_{2}$ hollow colloids and magnetic multifunctional particles. Advanced Materials, 2008, 20(10): 1853-1858.

[24] SUN Y, HU X, YU J, et al. Morphosynthesis of hierarchical $\mathrm{MoO}_{2}$ nanoarchitectures as a binder-free anode for lithium-ion batteries. Energy \& Environmental Science, 2011, 4: 2870-2877.

[25] WANG X, CHEN Z, LIU D, et al. Solar cells: triple-yolked $\mathrm{ZnO} / \mathrm{CdS}$ hollow spheres for semiconductor-sensitized solar cells. Particle \& Particle Systems Characterization, 2014, 31(7): 757-762.

[26] MAO R, GUO H, TIAN D X, et al. Hollow nanotubular $\mathrm{SnO}_{2}$ templated by cellulose fibers for lithium ion batteries. Journal of Inorganic Materials, 2013, 28(11): 1213-1216.

[27] CAI L, RAO P M, ZHENG X. Morphology-controlled flame synthesis of single, branched, and flower-like $\alpha-\mathrm{MoO}_{3}$ nanobelt arrays. Nano Letters, 2011, 11(2): 872-877.

[28] RILEY L A, LEE S H, GEDVILIAS L, et al. Optimization of $\mathrm{MoO}_{3}$ nanoparticles as negative-electrode material in high-energy lithium ion batteries. Journal of Power Sources, 2010, 195(2): $588-592$. 